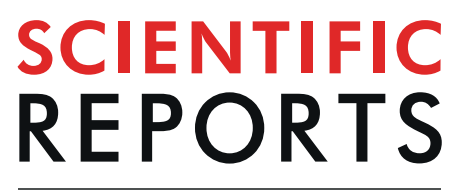

natureresearch

Check for updates

\title{
Influence of the long-term use of oral hygiene products containing stannous ions on the salivary microbiome - a randomized controlled trial
}

\begin{abstract}
A. C. Anderson ${ }^{1 凶}$, A. Al-Ahmad ${ }^{1}$, N. Schlueter ${ }^{2}$, C. Frese ${ }^{3}$, E. Hellwig ${ }^{1}$ \& N. Binder ${ }^{4,5}$
Oral hygiene products containing tin are suitable to prevent erosive tooth wear, yet effects on the oral microbiota are not known yet. Therefore, this study determined the salivary microbiome of 16 participants using products with stannous ions for three years (TG) compared with a control group (CG) to assess their influence on the microbiota. Participants were included in a randomized controlled clinical trial (RCT) with biannual visits. Illumina Miseq sequencing revealed as most abundant genera: Streptococcus (TG 14.3\%; CG 13.0\%), Veillonella (TG 11.3\%; CG 10.9\%), Prevotella (TG 7.0\%; CG 9.8\%), Haemophilus (TG 6.6\%; CG 7.2\%), Porphyromonas (TG 5.9\%, CG 5.1\%), Leptotrichia (TG 5.8\%; CG 4.9\%), Actinomyces (TG 4.0\%; CG 4.6\%) and Neisseria (TG 5.4\%; CG 4.2\%). Beta-Diversity was not significantly different between groups at both time points, although significant differences between groups were found for certain taxa after three years. The genus Prevotella was found in higher abundance in CG whereas Neisseria and Granulicatella, health-associated taxa, were found more abundantly in TG. Salivary microbiota after three years reflected a composition associated with oral health, hence continual use as a preventive measure for dental erosion can be considered safe and benefitting oral health for patients with a high risk of erosion.
\end{abstract}

Erosive tooth wear, a loss of dental hard tissue due to the direct impact of acids occurs worldwide with a prevalence of 20 to $40 \%{ }^{1}$. Diets high in acidic beverages and foods, gastro-esophageal reflux and eating disorders, e.g. bulimia, are among the factors that provoke this gradual loss of enamel and dentine ${ }^{2}$. The anti-erosive effect of stannous compounds has been studied in the last decades. Products containing stannous ions in combination with fluoride $(\mathrm{F} / \mathrm{Sn})$ have been found to exert marked anti-erosive effects, reducing tissue loss in acidic challenges in numerous studies ${ }^{3-6}$ and are considered standard ${ }^{7}$. The stannous compounds form a protective layer on the enamel surface and they can be incorporated into the enamel under erosive conditions protecting the underlying structures against erosive challenges ${ }^{8,9}$. This data emphasizes the erosion-preventive effect of these products. Oral hygiene products containing stannous ions also have an antibacterial effect and are therefore potentially beneficial concerning prevention of caries and periodontitis. To date many studies have analyzed the effect of stannous ions on selected microbial species or the general bacterial count, yet it has not been understood how these compounds influence the microbial community composition as a whole ${ }^{10-13}$. Furthermore, the long-term effect of these products on the oral microbiota in vivo has not been studied so far.

\footnotetext{
${ }^{1}$ Department of Operative Dentistry and Periodontology, Medical Center-University of Freiburg, Faculty of Medicine, University of Freiburg, Freiburg, Germany. ${ }^{2}$ Division for Cariology, Department of Operative Dentistry and Periodontology, Medical Center- University of Freiburg, Faculty of Medicine, University of Freiburg, Freiburg, Germany. ${ }^{3}$ Department of Conservative Dentistry, Clinic for Oral, Dental and Maxillofacial Diseases, University Hospital Heidelberg, Heidelberg, Germany. ${ }^{4}$ Institute for Prevention and Cancer Epidemiology, Faculty of Medicine and Medical Center, University of Freiburg, Freiburg, Germany. ${ }^{5}$ Institute of Digitalization in Medicine, Faculty of Medicine and Medical Center, University of Freiburg, Freiburg, Germany. ${ }^{\varpi_{e}}$-mail: annette.anderson@uniklinikfreiburg.de
} 


\begin{tabular}{|c|c|c|c|c|c|c|c|c|}
\hline \multirow{2}{*}{$\begin{array}{l}\text { Study } \\
\text { group }\end{array}$} & \multirow{2}{*}{\begin{tabular}{|l|}
$\begin{array}{l}\text { BMI }[\mathrm{kg} / \\
\left.\mathrm{m}^{2}\right]^{*}\end{array}$ \\
$\mathrm{t}=\mathbf{0}$
\end{tabular}} & \multirow{2}{*}{\begin{tabular}{|l|}
$\begin{array}{l}\text { Age } \\
\text { [years]* }\end{array}$ \\
$\mathbf{t}=0$ \\
\end{tabular}} & \multicolumn{2}{|l|}{ sex } & \multicolumn{2}{|c|}{ Saliva $\mathrm{pH}^{*}$} & \multicolumn{2}{|c|}{$\begin{array}{l}\text { Saliva flow rate } \\
{[\mathrm{ml} / \mathrm{min}]^{*}}\end{array}$} \\
\hline & & & Male & Female & $t=0$ & $t=3$ & $t=0$ & $t=3$ \\
\hline TG & $\begin{array}{l}23.06 \\
\pm 2.59 \\
\end{array}$ & \begin{tabular}{|l|}
38.0 \\
\pm 10.85 \\
\end{tabular} & $12(75 \%)$ & $4(25 \%)$ & \begin{tabular}{|l|}
7.05 \\
\pm 0.69 \\
\end{tabular} & \begin{tabular}{|l|}
6.76 \\
\pm 0.32 \\
\end{tabular} & \begin{tabular}{|l|}
2.33 \\
\pm 0.97 \\
\end{tabular} & \begin{tabular}{l|}
1.98 \\
\pm 0.70
\end{tabular} \\
\hline CG & $\begin{array}{l}23.01 \\
\pm 2.35\end{array}$ & \begin{tabular}{|l|}
38.7 \\
\pm 9.50
\end{tabular} & $19(86 \%)$ & $3(13 \%)$ & \begin{tabular}{|l|}
6.83 \\
\pm 1.19
\end{tabular} & \begin{tabular}{|l|}
6.72 \\
\pm 0.36
\end{tabular} & \begin{tabular}{|l|}
2.15 \\
\pm 0.90
\end{tabular} & $\begin{array}{l}1.87 \\
\pm 0.82\end{array}$ \\
\hline
\end{tabular}

Table 1. Demographic and clinical data of study participants $(n=38)$ at baseline and after three years $(t=0$, $\mathrm{t}=3)$. (Data modified from $\left.{ }^{33}\right) *($ mean $\pm \mathrm{SD})$.

Salivary microbiota, similar to other oral niches, contain commensal microorganisms that -in health- comprise a physiological flora that is beneficial as long as homeostasis is maintained ${ }^{14}$. Environmental disturbances can increase the abundance of potentially pathogenic species leading to oral diseases, such as caries and periodontitis $^{15,16}$. Therefore excessive eradication of the resident flora is not expedient, but it would be desirable to selectively influence the proportion of potentially pathogenic species ${ }^{17}$. In recent years, the salivary microbiome has been studied more extensively. The findings suggest a salivary core microbiome being present in the majority of individuals, accounting for a very large proportion of determined sequences and being fairly stable temporally ${ }^{18-20}$. The salivary microbiota resemble various oral sites, e.g. the tongue, the supragingival as well as subgingival plaque and are thought to be shaped by microorganisms deriving from these niches ${ }^{21}$. Certain taxa in saliva have been associated with specific oral diseases, e.g. periodontitis, early childhood caries or with oral health ${ }^{21-23}$. Therefore, besides investigating the clinical efficacy of oral hygiene products containing stannous ions, it is essential to investigate their impact on the microbial community. Hence, the aim of our study was to analyze the influence of the long-term use of a mouthrinse and toothpaste containing $\mathrm{SnCl}_{2} / \mathrm{AmF} / \mathrm{NaF}$ over three years on the salivary microbiome of an intervention group compared to a control group using products containing AmF/ $\mathrm{NaF}$ alone. To achieve this objective, we applied high-throughput DNA amplicon sequencing based on the $16 \mathrm{~S}$ rDNA on the Illumina MiSeq platform.

\section{Results}

Participant data. Fifty-four volunteers were recruited, 41 male and 13 female (mean age $36.53 \pm 9.49$ years; range 20-60 years), 27 for each group. The overall dropout rate was $29.6 \%$ with 5 subjects resigning from the control, and 11 subjects from the test group. Table 1 gives the summarized demographic and clinical parameters; detailed data are given in Supplementary Table S1. Supplementary Fig. S1 shows the flow chart according to CONSORT.

Composition of the salivary microbiota in TG and CG. Illumina Miseq sequencing of the 38 remaining saliva samples at T3 (three years) achieved an average of 120,649 raw reads per sample which resulted in an average 29,106 reads per sample after trimming, merging and quality filtering (Zymo Research Data). Taxonomic annotation based on Zymo Research Database (Zymoresearch, USA) using Uclust from Qiime (v.1.9.1, Caporaso et al. 2010) resulted in 285 different bacterial species belonging to 81 genera and 11 phyla. The most abundant phyla were Firmicutes (TG 43.7\%; CG 39.5\%), Bacteroidetes (TG 18.9\%, CG 24.1\%; significantly higher in CG; $\mathrm{p}=0.045$ ), Proteobacteria (TG 18.8\%; CG 18.3\%), Actinobacteria (TG 8.4\%; CG 8.0\%) and Fusobacteria (TG 7.6\%; CG 7.4\%) as depicted in Fig. 1a and Supplementary Fig. S2. For comparison, the DADA2 software pipeline based on Silva database v132 was used and resulted in 89 different bacterial genera belonging to 10 phyla. The most abundant phyla found in both groups were Firmicutes (TG 41.6\%; CG 38.8\%), Bacteroidetes (TG 20.4\%; CG 24.3\%), Proteobacteria (TG 14.0\%; CG 13.7\%), Actinobacteria (TG 9.6\%; CG 9.65\%) and Fusobacteria (TG 9.1\%; CG 8.0\%). The most abundant genera detected were Streptococcus (TG 14.3\%; CG 13.0\%), followed by Veillonella (TG 11.3\%; CG 10.9\%), Prevotella (TG 7.0\%; CG 9.8\%), Haemophilus (TG 6.6\%; CG 7.2\%), Porphyromonas (TG 5.9\%, CG 5.1\%), Leptotrichia (TG 5.8\%; CG 4.9\%), Actinomyces (TG 4.0\%; CG 4.6\%) and Neisseria (TG 5.4\%; CG 4.2\%). Figure 1b,c shows the abundances for CG and TG. The alpha diversity between TG and CG did not differ, but the species richness was significantly lower in TG, (TG 90 obs. sp., CG 106.8; p =0.047; based on Zymo Research data; Supplementary Table S2) At T0 (baseline), the same most abundant phyla and genera were detected in both groups (depicted in Supplementary Figs. S3-S5). The full list of OTUs and ASVs and their relative abundances are given in Supplementary Table 4 and 5. In the following we will focus on the results based on the Zymo Research Database.

In order to assess the differences between the salivary microbiome of the two groups, the beta-diversity was analyzed on the basis of the Bray-Curtis dissimilarity. The non-metric multidimensional scaling did not show any distinct clustering in either TG or CG at T0 nor at T3, and there were no significant differences in the calculated beta-diversity (permutational multivariate analysis of variance based on Bray-Curtis dissimilarity; $\mathrm{p}=0.447$ at T0; $\mathrm{p}=0.302$ at T3.) (Fig. 2). Consequently, the salivary bacterial community as a whole is not significantly affected by the long-term use of products containing $\mathrm{SnCl} 2 / \mathrm{AmF} / \mathrm{NaF}$.

Even though there was no significant difference in beta-diversity, certain taxa showed a significantly different abundance in the two groups at T3, but not at T0. A LEfSe analysis revealed that Prevotella taxa, $P$. histicola jejuni_veroralis, $P$. salivae and P. loescheii as well as Eikenella sp., Kingella oralis and Haemophilus haemolyticus were found in significantly higher abundance in CG. Neisseria sp., Granulicatella sp., Gemella sanguinis and Kingella denitrificans were found enriched in TG (Fig. 3). Separate Wilcoxon tests confirmed this, showing a significantly higher abundance of the genus Prevotella in CG $(\mathrm{p}=0.022)$ and a significantly higher abundance of 
a

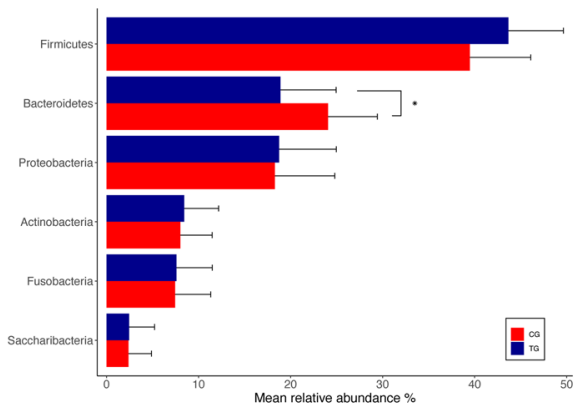

d

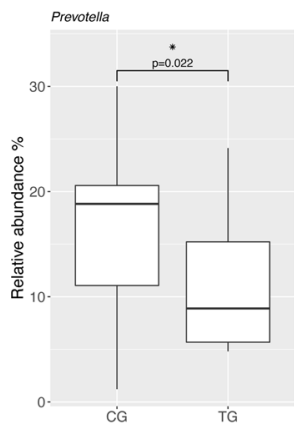

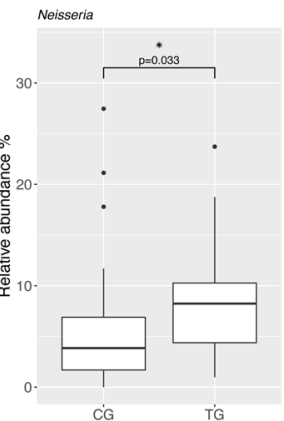

CG b

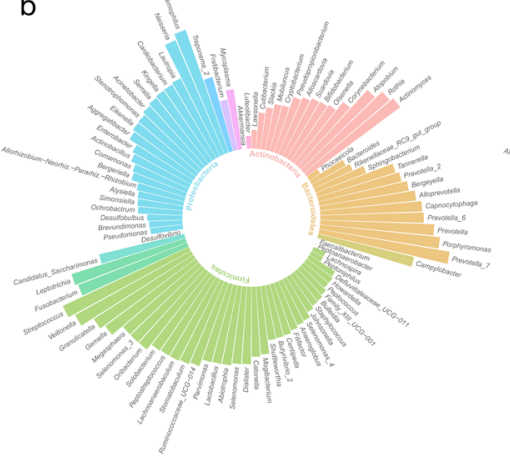

e

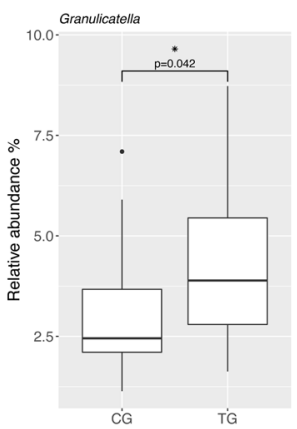

C

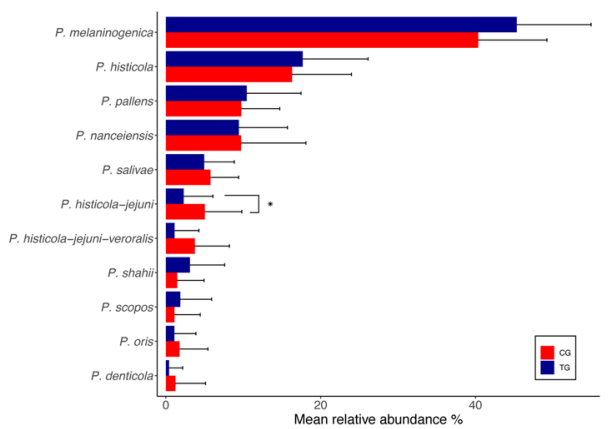

Figure 1. (a) Relative abundances of the top six phyla ( $>1 \%$ abundance) detected in saliva in TG (Treatment group, blue) and CG (Control group, red) $(* p \leq 0.05)(\mathbf{b})$ Relative abundance of the bacterial genera detected in saliva in CG, (c) Relative abundance of the bacterial genera detected in saliva in TG, (d) Relative abundances of the genera Prevotella, Neisseria and Granulicatella in CG and TG, (e) Relative abundances of the different Prevotella species ( $>1 \%$ abundance) in TG (blue) and CG (red), $(* \mathrm{p} \leq 0.05)$.

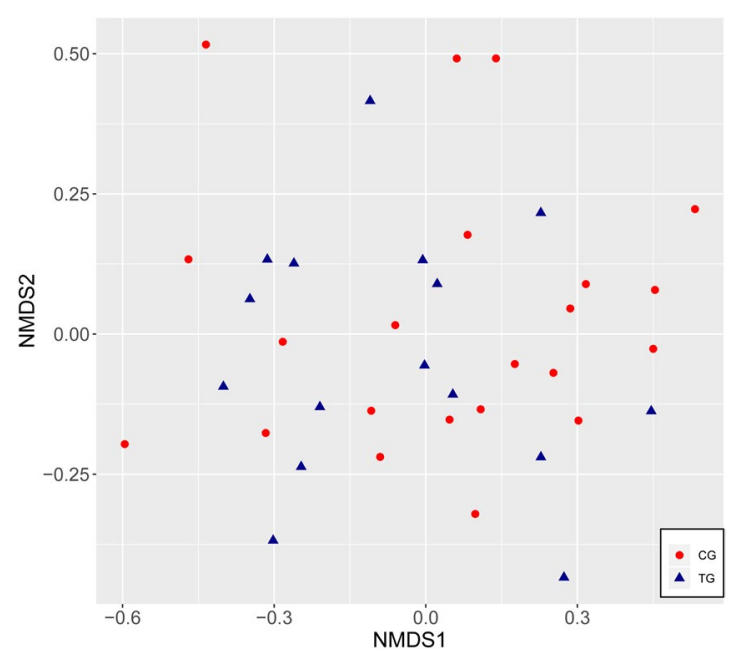

Figure 2. NMDS plot (non-metric multidimensional scaling) depicting the beta-diversity of the microbiota in saliva in CG and TG (Zymo Research Data).

the genus Neisseria $(\mathrm{p}=0.033)$ as well as Granulicatella $(\mathrm{p}=0.042)$ in TG (Fig. 1d). The analysis of the sequences on the species level revealed that certain Prevotella species were more abundant in CG (e.g. P. histicola_jejuni $\mathrm{p}=0.041$; P. histicola_jejuni_veroralis, n.s.) (Fig. 1e; Supplementary Fig. S6). At baseline, these taxa did not show any significant differences (Supplementary Fig. S7). When the abundance of Prevotella species was related to the distribution of males and females in the two groups, significant gender-related differences were revealed (Fig. 4). Thus, 2 Prevotella taxa were significantly more abundant in males (e.g. P. histicola_jejuni, $\mathrm{p}=0.035 ;$ P. histicola_ jejuni_veroralis $\mathrm{p}=0.015)$. The genera Neissera and Granulicatella did not show any gender-related differences in their abundance. 


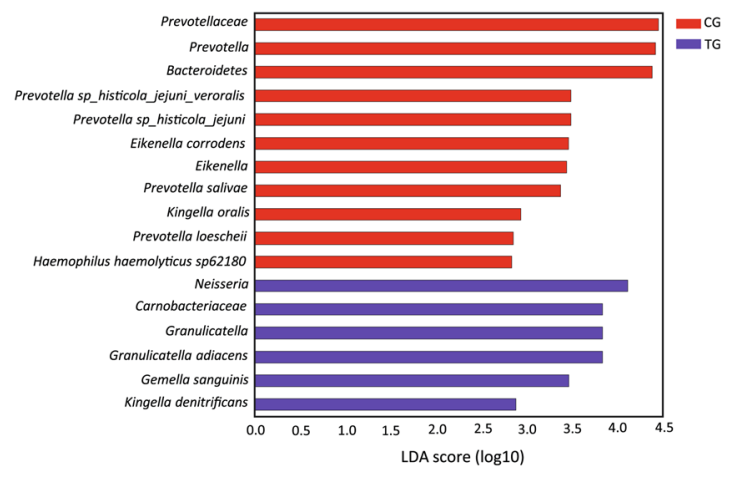

Figure 3. Bar graph of LEfSe analysis of bacterial taxa in saliva in CG (red) and TG (blue) showing LDA scores (Biomarkers sorted according to effect size).

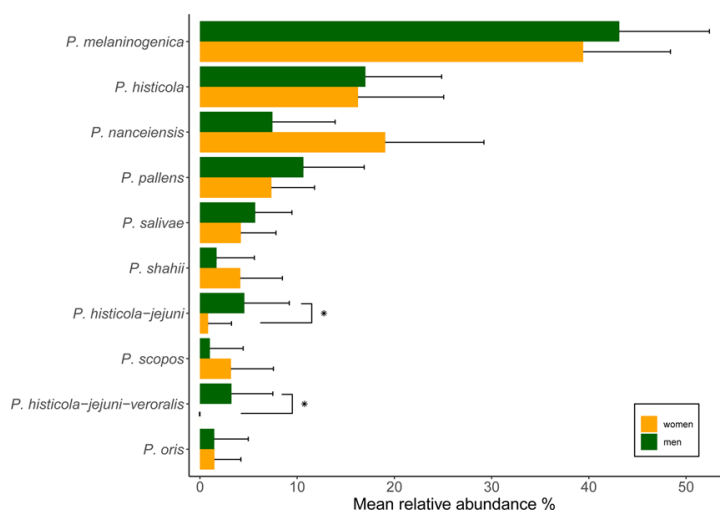

Figure 4. Relative abundances of the different Prevotella species ( $>1 \%$ abundance) in saliva of both groups related to the gender of the study participants (green $=$ men, orange $=$ women), $\left({ }^{*} \mathrm{p} \leq 0.05\right)$.

As far as the mock community used as positive control, the Illumina MiSeq sequencing detected all taxa included in the mock community. Contaminant reads were $\leq 5$ reads per sequenced sample. The relative abundances of the included taxa were not equal, Tannerella sp. and the two Streptococcus species, which were not differentiated (S. mitis and S. sanguinis) were underrepresented and on the other hand Porphyromonas sp. and Fusobacterium s.p. were somewhat overrepresented. The results of the mock community sequencing are shown in Supplementary Table S3.

\section{Discussion}

Oral hygiene products containing stannous ions are suitable for continuous use as preventive measures ${ }^{24}$, however, their long-term influence on the oral microbiota has not been studied yet. Therefore, this study aimed to analyze the salivary microbiome of individuals with elevated risk for dental erosion that used products containing $\mathrm{SnCl}_{2} / \mathrm{AmF} / \mathrm{NaF}$ over a period of three years in comparison to a control group. Since the salivary microbiota contains bacteria shed from different oral sites its composition is thought to reflect the oral microbiota as a whole ${ }^{21,25}$. Therefore there is an increased interest in sampling saliva to determine oral health, and to our knowledge this is the first study that investigated the effect of a long-term use of such products on the salivary microbiota in vivo. The most noteworthy outcome was that no significant difference could be observed in the microbial composition in TG compared to CG at both time points, even though certain taxa showed significantly different abundances in the two groups at T3. Overall, the microbiome of TG after the three-year treatment period revealed a composition of microbial taxa that can be interpreted as health-associated.

Previous studies have proven the ability of stannous ions to reduce plaque and salivary bacterial count. Addy et al. tested their effect on the salivary bacterial count and on plaque regrowth and reported a higher reduction compared to the control ${ }^{10}$. Auschill et al. evaluated the influence on the biofilm thickness of plaque and the bacterial viability and reported significant reduction of both parameters compared to the control ${ }^{26}$. Other authors tested the effect in vitro against single species or artificial biofilms consisting of certain oral taxa, e.g. Porphyromonas gingivalis, Fusobacterium nucleatum, Streptococcus mutans, S. sanguinis and reported the efficacy of these compounds to reduce bacterial growth ${ }^{11,13,27}$. Cheng et al. found growth of $S$. mutans and P. gingivalis suppressed whereas $S$. sanguinis was enriched after five days. Shapiro et al. found differentially reduced growth for several species in a six-species biofilm, with F. nucleatum and S. oralis reduced the most ${ }^{13}$.

However, in contrast to our study, the focus of these authors was the short-term effect of these products to reduce the bacterial load until plaque regrowth occurred. They failed to take into account the microbial 
community as a whole in vivo reacting to outward influences as an ecological framework with specific interactions between the taxa present. Our study used comprehensive high-throughput sequencing revealing the influence of the long-term use of stannous chloride on the whole microbial community. The samples of both groups, TG and CG revealed a high abundance of the genera Streptococcus, Veillonella, Prevotella, Granulicatella, Gemella, Haemophilus, Neisseria, Porphyromonas, Actinomyces and Rothia, corresponding to the taxa found as salivary core microbiome in many recent studies ${ }^{20,28,29}$. Regarding the species diversity, both groups did not differ from each other at T3 which is indicative that the treatment groups' microbiota represents a healthy status. Kumar et al. argue that similar levels of diversity are important, since any deviation from the controlled diversity in the oral ecosystem associated with a healthy status appears to result in a dysbiotic condition, eventually leading to disease $^{30}$. On the other hand, the species richness was lower in TG, which is also indicative of a healthy status ${ }^{20,31}$.

As for differentially abundant taxa found in the two groups at T3, the genera Prevotella, Neisseria and Granulicatella stand out. The genus Prevotella was found in significantly higher abundances in CG (Fig. 1d). In part this could be attributed to the fact that the control group included more men and $P$. histicola-jejuni and $P$. histicola-jejuni-veroralis were significantly more abundant in males. Zaura et al., analyzing the salivary microbiome of 268 individuals also reported a higher abundance of Prevotella spp. in males, whereas females showed a higher abundance of certain Streptococcus species ${ }^{29}$. Yet, since the whole genus Prevotella, showed a significantly higher abundance in CG it can be assumed that stannous chloride might suppress the growth of many representatives of this taxon.

Interestingly, Yang et al., studying salivary microbiomes of caries-active and healthy individuals, found an association of Prevotella sp. (P. histicola among others) with caries-active individuals which was confirmed by Teng et al., who reported an increased abundance of various Prevotella species in the saliva of children with early childhood caries ${ }^{22,23}$. According to their assumption, this taxon could be indicative of a caries-active status. Belstrom et al., comparing saliva of healthy individuals with caries and periodontitis patients, showed that traditional periodontal pathogens and cariogenic species, were found significantly more often in the saliva from patients with the respective condition ${ }^{21}$. As for Prevotella taxa, $P$. intermedia, P. baroniae and $P$. falsenii were enriched in periodontitis patients, $P$. denticola in caries patients, yet $P$. scopos and $P$. bergensis were associated with oral health. Regarding the genera Neisseria and Granulicatella being more abundant in TG, both taxa can be regarded as health-associated. Yamashita et al. were able to differentiate several types of salivary microbiomes according to periodontal health and found a high abundance of Neisseria flavescens, Granulicatella adiacens and some other taxa enriched in periodontally healthy individuals. On the other hand, individuals with a high abundance of P. histicola, P. melaninogenica, several Veillonella and Streptococcus species exhibited poorer oral health ${ }^{31}$. Correspondingly, Sanz et al. infer from recent high-throughput sequencing studies that Neisseria and Granulicatella among other taxa can be regarded as genera normally associated with oral health ${ }^{32}$. Taken all together, we can conclude that the differential abundances of the genera Prevotella, Neisseria and Granulicatella, i.e. a lower abundance of Prevotella spp. and a higher abundance of the latter two after the long-term use of products containing stannous ions can be seen as a beneficial effect for the entirety of the oral microbiota.

In conclusion, the analysis of the salivary microbiome after a long-term use of oral hygiene products containing $\mathrm{SnCl}_{2} / \mathrm{AmF} / \mathrm{NaF}$ reflects a healthier status of the microbial community. This result was also confirmed by the clinical data on the oral health status demonstrated by the biannual caries assessment by the ICDAS-II-System for both groups ${ }^{33}$. The clinical data on erosion showed that the treatment group yielded a better result after four years with less increment in erosion ${ }^{34}$. In this respect the continual use of these products as a preventive measure for tooth erosion can be recommended for patients with a high risk of erosion and can be considered as contributing to oral health in general.

\section{Materials and Methods}

Study design. The study was performed as an RCT in accordance with guidelines of Good Clinical Practice and conforming to the declaration of Helsinki. Ethical approval was obtained from the local ethics committee (S-566/2012; University of Heidelberg). The study was registered at the German Clinical Trials Registry Platform (DRKS00005019, registration 2013/05/27). Previous studies have shown that endurance athletes are at a higher risk of erosive tooth wear than controls ${ }^{34}$. For this reason, 54 participants who performed at least five hours of endurance training per week were recruited between March and October 2013 and included in the study. Inclusion criteria were: good general health, written informed consent. Exclusion criteria were: cumulative weekly training under five hours, restrictions in oral hygiene, pregnancy or lactation, participation in another clinical study within the last 30 days, use of antibiotics within the last 30 days prior to the study and each recall time point, affiliation with the dentistry department as student or staff. The full details of the study are given elsewhere $^{33}$. Randomization of the participants into treatment group (TG) and control group (CG) was performed by block randomization (using sequentially numbered envelopes). The numbers of participants withdrawing from the study were documented in the CONSORT flow diagram (Fig. S1).

Clinical oral examinations were performed every 6 months with assessment of dental erosion (BEWE), caries index (ICDAS II) and a professional tooth cleaning at the Department of Conservative Dentistry, University Hospital Heidelberg (Heidelberg, Germany) by one blinded calibrated examiner ${ }^{33,34}$. For the analysis of the salivary microbiota stimulated saliva was sampled from each participant at baseline and after the three-year treatment period using sterile paraffin gum. Each participant refrained from eating and drinking, smoking and brushing their teeth for at least 2 hours before sampling.

The participants in the TG received mouth rinse containing F/Sn $\left(\mathrm{AmF} / \mathrm{NaF} / \mathrm{SnCl}_{2} ; 800 \mathrm{ppm} \mathrm{Sn}{ }^{2+}, 500 \mathrm{ppm}\right.$ F-; Elmex Erosion Protection Mouthrinse, CP GABA, Hamburg, Germany) and toothpaste containing F/Sn (NaF/ $\mathrm{Sn}^{2+} ; 3500$ ppm Sn ${ }^{2+}, 1450$ ppm F${ }^{-}$; Elmex Erosion Protection Toothpaste; CP GABA, Hamburg, Germany) and were instructed to use mouthrinse $1 \times 30$ s per day and toothpaste twice a day for routine oral hygiene. The participants in the CG did not receive any oral hygiene products but were given instructions to use conventional 
fluoridated toothpaste (1500 ppm) but no products containing stannous compounds. During the follow-up visits the compliance of the participants of both groups was verified, the brands of the oral hygiene products used by the CG were retrieved and all the instructions were repeated.

16S rDNA Illumina MiSeq high-throughput sequencing. To analyze the salivary microbiome we applied high-throughput DNA amplicon sequencing based on the 16S rDNA on the Illumina MiSeq platform. For DNA extraction the DNA PowerSoil Kit (Qiagen, Hilden, Germany) was used according to the manufacturer's protocol. The microbial community was analyzed using Illumina MiSeq paired-end-sequencing with two times $300 \mathrm{bp}$ read length. The amplicon library was constructed using the primers 5'-CCTACGGGNGGCWGCAG-3' and $5^{\prime}$-GACTACHVGGGTATCTAATCC- $3^{\prime 35}$ for the variable regions v3-v4 including the recommended adaptors for Illumina Sequencing (MiSeq Reagent Kit v3, Illumina, Eindhoven, Netherlands). Amplification, indexing, library quantification, pooling and sequencing were performed according to the Illumina MiSeq protocol for amplicon sequencing. Filtering, denoising, merging and the taxonomic classification based on the Silva database (v132 ${ }^{36}$; was done with the Divisive Amplicon Denoising Algorithm 2 (DADA2) pipeline ${ }^{37}$. For comparison, taxonomic annotation was performed using Uclust (Qiime v. 1.9.1) based on a different database (Zymo Research, Irvine, USA) to achieve further taxonomic assignment on the species level.

Mock community. As a positive control we created an internal mock community containing the following microbial species in equal proportions as measured by $\mathrm{OD}_{600}$ : Streptococcus sanguinis (DSM 20068), Streptococcus mitis (ATCC11843), Porphyromonas gingivalis (W381), Fusobacterium nucleatum (ATCC 25586) and Tannerella forsythia (ATCC 43037). The DNA of this mixture was extracted using the DNA PowerSoil Kit according to the manufacturer's protocol and was included in the Illumina MiSeq sequencing procedure.

Statistical analysis. All statistical analyses were performed in R (V.3.6.0). As taxa abundances are not normally distributed, non-parametric tests (Wilcoxon Rank Sum Test) were used with multiple testing correction when applicable. P-values $<0.05$ were regarded as statistically significant. The species richness was determined using Chaol (richness) and alpha diversity was analyzed using Shannon Diversity (OTU-based diversity). Beta diversity was assessed by computing weighted Bray-Curtis distances to compare microbial communities based on relative abundance for each microbial community. NMDS was performed to compare beta diversity among groups. To determine features most likely to explain differences between TG and CG, we applied the linear discriminant analysis (LDA) effect size (LEfSe) method for differential abundance analysis ${ }^{38}$.

\section{Data availability}

The datasets supporting the conclusion of this article are available through GenBank (SRA accession: PRJNA577839; SUB6429482; accession numbers SRX7007447 - SRX7007521 and can be found at https://www. ncbi.nlm.nih.gov/sra/PRJNA577839 after the release date.

Received: 18 November 2019; Accepted: 19 May 2020;

Published online: 12 June 2020

\section{References}

1. Schlueter, N. \& Luka, B. Erosive tooth wear - a review on global prevalence and on its prevalence in risk groups. Br Dent J 224, 364-370, https://doi.org/10.1038/sj.bdj.2018.167 (2018).

2. Ganss, C., Lussi, A. \& Schlueter, N. Dental erosion as oral disease. Insights in etiological factors and pathomechanisms, and current strategies for prevention and therapy. Am J Dent 25, 351-364 (2012).

3. Ganss, C., Neutard, L., von Hinckeldey, J., Klimek, J. \& Schlueter, N. Efficacy of a tin/fluoride rinse: a randomized in situ trial on erosion. J Dent Res 89, 1214-1218, https://doi.org/10.1177/0022034510375291 (2010).

4. Joao-Souza, S. H. et al. In situ evaluation of fluoride-, stannous- and polyphosphate-containing solutions against enamel erosion. $J$ Dent 63, 30-35, https://doi.org/10.1016/j.jdent.2017.05.014 (2017).

5. Rakhmatullina, E., Beyeler, B. \& Lussi, A. Inhibition of enamel erosion by stannous and fluoride containing rinsing solutions. Schweiz Monatsschr Zahnmed 123, 192-198 (2013).

6. Schlueter, N., Klimek, J. \& Ganss, C. In vitro efficacy of experimental tin- and fluoride-containing mouth rinses as anti-erosive agents in enamel. J Dent 37, 944-948, https://doi.org/10.1016/j.jdent.2009.07.010 (2009).

7. Carvalho, T. S. et al. Consensus report of the European Federation of Conservative Dentistry: erosive tooth wear-diagnosis and management. Clin Oral Investig 19, 1557-1561, https://doi.org/10.1007/s00784-015-1511-7 (2015).

8. Lussi, A. et al. The use of fluoride for the prevention of dental erosion and erosive tooth wear in children and adolescents. Eur Arch Paediatr Dent https://doi.org/10.1007/s40368-019-00420-0 (2019).

9. Schlueter, N. et al. Tin-containing fluoride solutions as anti-erosive agents in enamel: an in vitro tin-uptake, tissue-loss, and scanning electron micrograph study. Eur J Oral Sci 117, 427-434, https://doi.org/10.1111/j.1600-0722.2009.00647.x (2009).

10. Addy, M., Greenman, J., Renton-Harper, P., Newcombe, R. \& Doherty, F. Studies on stannous fluoride toothpaste and gel (2). Effects on salivary bacterial counts and plaque regrowth in vivo. J Clin Periodontol 24, 86-91 (1997).

11. Cheng, X. et al. Comparative effect of a stannous fluoride toothpaste and a sodium fluoride toothpaste on a multispecies biofilm. Arch Oral Biol 74, 5-11, https://doi.org/10.1016/j.archoralbio.2016.10.030 (2017).

12. Meurman, J. H., Kari, K., Aikas, A. \& Kallio, P. One-year compliance and effects of amine and stannous fluoride on some salivary biochemical constituents and oral microbes in institutionalized elderly. Spec Care Dentist 21, 32-36 (2001).

13. Shapiro, S., Giertsen, E. \& Guggenheim, B. An in vitro oral biofilm model for comparing the efficacy of antimicrobial mouthrinses. Caries Res 36, 93-100, https://doi.org/10.1159/000057866 (2002).

14. Marsh, P. D. In Sickness and in Health-What Does the Oral Microbiome Mean to Us? An Ecological Perspective. Adv Dent Res 29, 60-65, https://doi.org/10.1177/0022034517735295 (2018).

15. Anderson, A. C. et al. In-vivo shift of the microbiota in oral biofilm in response to frequent sucrose consumption. 8, 14202, https:// doi.org/10.1038/s41598-018-32544-6 (2018).

16. Sultan, A. S. \& Kong, E. F. The oral microbiome: A Lesson in coexistence. 14, e1006719, https://doi.org/10.1371/journal. ppat.1006719 (2018).

17. ten Cate, J. M. \& Zaura, E. The numerous microbial species in oral biofilms: how could antibacterial therapy be effective? Adv Dent Res 24, 108-111, https://doi.org/10.1177/0022034512450028 (2012). 
18. Cabral, D. J. et al. The salivary microbiome is consistent between subjects and resistant to impacts of short-term hospitalization. Sci Rep 7, 11040, https://doi.org/10.1038/s41598-017-11427-2 (2017).

19. Hall, M. W. et al. Inter-personal diversity and temporal dynamics of dental, tongue, and salivary microbiota in the healthy oral cavity. NPJ Biofilms Microbiomes 3, 2, https://doi.org/10.1038/s41522-016-0011-0 (2017).

20. Takeshita, T. et al. Bacterial diversity in saliva and oral health-related conditions: the Hisayama Study. Sci Rep 6, 22164, https://doi. org/10.1038/srep22164 (2016).

21. Belstrom, D., Constancias, F., Liu, Y. \& Yang, L. Metagenomic and metatranscriptomic analysis of saliva reveals disease-associated microbiota in patients with periodontitis and dental caries. 3, 23, https://doi.org/10.1038/s41522-017-0031-4 (2017).

22. Teng, F. et al. Prediction of Early Childhood Caries via Spatial-Temporal Variations of Oral Microbiota. Cell Host Microbe 18, 296-306, https://doi.org/10.1016/j.chom.2015.08.005 (2015).

23. Yang, F. et al. Saliva microbiomes distinguish caries-active from healthy human populations. Isme j 6, 1-10, https://doi.org/10.1038/ ismej.2011.71 (2012).

24. Huysmans, M. C., Young, A. \& Ganss, C. The role of fluoride in erosion therapy. Monogr Oral Sci 25, 230-243, https://doi. org/10.1159/000360555 (2014).

25. Kageyama, S. et al. Relative abundance of total subgingival plaque-specific bacteria in salivary microbiota reflects the overall periodontal condition in patients with periodontitis. 12, e0174782, https://doi.org/10.1371/journal.pone.0174782 (2017).

26. Auschill, T. M. et al. Effect of two antimicrobial agents on early in situ biofilm formation. J Clin Periodontol 32, 147-152, https://doi. org/10.1111/j.1600-051X.2005.00650.x (2005).

27. Schaeken, M. J., De Jong, M. H., Franken, H. C. \& Van der Hoeven, J. S. Effects of highly concentrated stannous fluoride and chlorhexidine regimes on human dental plaque flora. J Dent Res 65, 57-61, https://doi.org/10.1177/00220345860650011001 (1986).

28. Lazarevic, V., Whiteson, K., Hernandez, D., Francois, P. \& Schrenzel, J. Study of inter- and intra-individual variations in the salivary microbiota. BMC Genomics 11, 523, https://doi.org/10.1186/1471-2164-11-523 (2010).

29. Zaura, E., Brandt, B. W. \& Prodan, A. On the ecosystemic network of saliva in healthy young adults. 11, 1218-1231, https://doi. org/10.1038/ismej.2016.199 (2017).

30. Kumar, P. S. \& Mason, M. R. Mouthguards: does the indigenous microbiome play a role in maintaining oral health? Front Cell Infect Microbiol 5, 35, https://doi.org/10.3389/fcimb.2015.00035 (2015)

31. Yamashita, Y. \& Takeshita, T. The oral microbiome and human health. J Oral Sci 59, 201-206, https://doi.org/10.2334/ josnusd.16-0856 (2017)

32. Sanz, M. et al. Role of microbial biofilms in the maintenance of oral health and in the development of dental caries and periodontal diseases. Consensus report of group 1 of the Joint EFP/ORCA workshop on the boundaries between caries and periodontal disease. J Clin Periodontol 44(Suppl 18), S5-s11, https://doi.org/10.1111/jcpe.12682 (2017).

33. Frese, C. et al. Clinical management and prevention of dental caries in athletes: A four-year randomized controlled clinical trial. Sci Rep 8, 16991, https://doi.org/10.1038/s41598-018-34777-x (2018).

34. Frese, C. et al. Clinical effect of stannous fluoride and amine fluoride containing oral hygiene products: A 4-year randomized controlled pilot study. Sci Rep 9, 7681, https://doi.org/10.1038/s41598-019-44164-9 (2019).

35. Klindworth, A. et al. Evaluation of general $16 \mathrm{~S}$ ribosomal RNA gene PCR primers for classical and next-generation sequencingbased diversity studies. Nucleic Acids Res 41, e1, https://doi.org/10.1093/nar/gks808 (2013).

36. Quast, C. et al. The SILVA ribosomal RNA gene database project: improved data processing and web-based tools. Nucleic Acids Res 41, D590-596, https://doi.org/10.1093/nar/gks1219 (2013).

37. Callahan, B. J. et al. DADA2: High-resolution sample inference from Illumina amplicon data. Nat Methods 13, 581-583, https://doi. org/10.1038/nmeth.3869 (2016).

38. Segata, N. et al. Metagenomic biomarker discovery and explanation. Genome Biol 12(6), R60, https://doi.org/10.1186/gb-2011-126-r60 (2011).

\section{Acknowledgements}

The authors thank Bettina Spitzmüller for her excellent technical assistance and Grant Anderson for English language correction. We acknowledge financial support by the science fund of the Deutsche Gesellschaft für Zahn-, Mund- und Kieferheilkunde (DGZMK) for the experimental part of the high-throughput sequencing analysis and the provision of the test products (oral hygiene products) for the study participants by CP GABA $\mathrm{GmbH}$ (Hamburg, Germany). The article processing charge was funded by the Baden-Wuerttemberg Ministry of Science, Research and Art and the University of Freiburg in the funding programme Open Access Publishing.

\section{Author contributions}

N.S. and A.A.A. contributed to conception, design, data interpretation and critically revised the manuscript. A.C.A. and N.B. contributed to design, data analysis and interpretation, preparation of the figures and drafted and critically revised the manuscript. C.F. and E.H. contributed to data interpretation and critically revised the manuscript. All authors approved the final manuscript.

\section{Competing interests}

The authors declare no competing interests.

\section{Additional information}

Supplementary information is available for this paper at https://doi.org/10.1038/s41598-020-66412-z.

Correspondence and requests for materials should be addressed to A.C.A.

Reprints and permissions information is available at www.nature.com/reprints.

Publisher's note Springer Nature remains neutral with regard to jurisdictional claims in published maps and institutional affiliations. 
(c) (i) Open Access This article is licensed under a Creative Commons Attribution 4.0 International License, which permits use, sharing, adaptation, distribution and reproduction in any medium or format, as long as you give appropriate credit to the original author(s) and the source, provide a link to the Creative Commons license, and indicate if changes were made. The images or other third party material in this article are included in the article's Creative Commons license, unless indicated otherwise in a credit line to the material. If material is not included in the article's Creative Commons license and your intended use is not permitted by statutory regulation or exceeds the permitted use, you will need to obtain permission directly from the copyright holder. To view a copy of this license, visit http://creativecommons.org/licenses/by/4.0/.

(C) The Author(s) 2020 